\title{
How to Test Stringy Dark Energy?
}

\author{
P.H. Frampton \\ Department of Physics and Astronomy, University of North Carolina, Chapel Hill, NC 27599-3255.
}

It is suggested, by using a covariant lagrangian formalism to estimate the equation of state $w=p / \rho$, that stringy dark energy predicts $w<-1$, a negative pressure larger in magnitude than that for a cosmological constant or quintessence. This would lead to a later transition from decelerating to accelerating cosmological expansion; $w=-4 / 3$ is briefly considered as one illustrative example.

The last few years have witnessed a revolution in our knowledge of the universe. Paramount among recent observational discoveries the phenomenon of dark energy, probably the most surprising discovery in physics or astronomy since parity violation almost a half a century ago. As in that case, the explanation of dark energy will surely impact a wide range of disciplines.

It was recently proposed that the cosmological dark energy which causes the present accelerated expansion of the universe may arise from a stringy origin [1]. In particular, in a toroidal closed string universe the correlation between winding and momentum modes of closed strings leads in the phase transition at the Hagedorn temperature to a condensate phenomenon.

For stringy dark energy, the pressure can certainly be negative because for exponentially falling $\omega(\kappa)$ the group velocity $v_{g} \propto d \omega(\kappa) / d \kappa$ is negative and can dominate the pressure expression. The detailed calculation of the value of $w(Z)$ depends on the Hagedorn phase transition where the closed strings are strongly interacting and hence impracticable to compute. Still we can hope to test the approach already by some estimates. The idea of an exponentially decrease of $\omega(\kappa)$ at large transplanckian $\kappa$ providing a candidate for dark energy was proposed in [2].

We discuss the pressure estimate first in general terms followed by a more specific calculation. Finally, the resultant red-shift for the transition from decelerated to accelerated cosmological expansion is briefly dicussed.

General dispersion relation. Consider a spatially flat FRW universe with the spacetime line element,

$$
d s^{2}=-d t^{2}+a(t)^{2}(\overrightarrow{d x})^{2} .
$$

Let $\phi$ be a field satisfying a wave equation that, either exactly or in the adiabatic approximation, has plane wave solutions,

$$
\phi_{\vec{k}}(\vec{x}, t)=\exp \left[i\left(\vec{k} \cdot \vec{x}-\int^{t} \omega\left(k / a\left(t^{\prime}\right)\right) d t^{\prime}\right],\right.
$$

where $k \equiv|\vec{k}|$. If the wave equation were Lorentz covariant in the flat spacetime limit, then one would have $\omega\left(k / a\left(t^{\prime}\right)\right)=\left(k^{2} / a^{2}+m^{2}\right)^{1 / 2}$. We will suppose that $\omega$ has this form when the momentum $k / a(t)$ is small with respect to the Planck mass, $m_{*}$. For larger momenta, we will suppose that $\omega(c)$ is a decreasing function of $c \equiv k / a(t)$.

The group velocity, $v_{g}$, of a wave packet,

$$
\phi(x, t)=\int d^{3} k A(k) \phi_{\vec{k}}(\vec{x}, t)
$$

formed from these plane waves over a narrow range of $\vec{k}$ 's near the value $\vec{k}$, is

$$
\vec{v}_{g}(t) \equiv a(t) d \vec{x}_{\text {peak }}(t) / d t=\left(\nabla_{\vec{c}} \omega(c)\right)_{\vec{c}=\vec{k} / a(t)}
$$

It follows that the group velocity is negative if $k$ is larger than $m_{*}$. This means that the wave packet moves in the direction opposite to the momentum, $\vec{k} / a(t)$. Consider a set of such wave packets contained at time $\mathrm{t}$ within a given comoving volume. When one of the wave packets passes out of the comoving volume it transfers momentum into the volume. This means that the pressure exerted by the energy density inside a comoving volume on the bounding surface of the volume is negative. As in kinetic theory, one may calculate the momentum transfer per unit time and per unit area. This gives the pressure $P$ exerted by such wave packets as,

$$
P(t)=\frac{N}{3} \int d^{3} k n(c(t)) c(\vec{t}) \cdot \vec{v}_{g}(c(t))
$$

Here $n(c(t))$ is the number per unit physical volume, of wave packets having momentum $c(t)$ at time $t$. This number density is uniform at any given time. Similarly, one finds that the energy density $\rho$ of these wave packets is

$$
\rho(t)=N \int d^{3} k n(c(t)) \omega(c(t))
$$


The packets with momenta that satisfy $c(t)>m_{*}$ exert negative pressure, but have positive energy density. Therefore, they may contribute to the recent acceleration of the universe. Packets that satisfy this condition, correspond to modes with $k>m_{*} a(t)$. A mode, $\vec{k}$, that satisfies this condition at the present time, had to have transplanckian momenta in the very early universe. Since $\omega(c(t))$ decreases with increasing values of $c$ larger than $m_{*}$, the energy $\omega$ of such a packet was less in the early universe than it is today. This is consistent with negative pressure, which causes the energy density to increase as the universe expands. The pressure and energy density of these packets satisfies the local conservation law stated in Eq.(18) below.

A given mode $\vec{k}$ that has momentum $k / a(t) \gg$ $m_{*}$ will increase in energy $\omega(k / a(t))$ as the universe expands and will exert negative pressure until the momentum becomes less than $m_{*}$. After that, the energy and pressure of the mode behaves more like that of an ordinary particle.

The pressure and energy density here refer to the specific coordinate system in which the preferred geodesics correspond to world lines of constant $\vec{x}$. Thus, the meaning of transplanckian momentum is defined with respect to the set of preferred geodesics. Furthermore, $P$ and $\rho$ are the pressure and energy density that would be measured by an observer on one of these geodesics. Thus, they are scalar quantities. The corresponding energymomentum tensor for this set of packets is therefore

$$
T_{\mu \nu}=P g_{\mu \nu}+(\rho+P) u_{\mu} u_{\nu}
$$

Then Eq. (18) is equivalent to the local conservation law, $T^{\mu \nu}{ }_{i \nu}=0$, for the metric of Eq. (11), as well as in any spatially curved FRW universe.

The extension of this discussion to spatially curved FRW universes is straightforward, with the spatial part of the mode functions, $\exp (i \vec{k} \cdot \vec{x})$, replaced by the appropriate harmonic functions that are eigenfunctions of the spatial Laplacian operator.

In an FRW spacetime having metric of Eq. (1), once the energy function $\omega$ is chosen, one can calculate its effect on the scale factor $a(t)$ through the Einstein equations,

$$
R_{\mu \nu}-(1 / 2) g_{\mu \nu} R=8 \pi G T_{\mu \nu}
$$

where now the energy-momentum tensor includes all contributions. The negative pressure coming from the modes with transplanckian momenta will tend to accelerate the expansion of the universe. The degree of acceleration will depend the density and pressure of other forms of matter present and on the rate at which $\omega$ decreases with increasing momentum in the transplanckian range. One simple example of a possible $\omega$ is

$$
\omega(c)=\sqrt{\vec{c}^{2}+m^{2}} \exp \left(-c / m_{*}\right),
$$

where $\mathrm{m}$ is the mass of the field $\phi$ and $m_{*}$ is the Planck mass. In the next section, we discuss a Lagrangian which gives rise to a particular dispersion relation $\omega$ that has a form similar to Eq. (9), but with $\exp \left(-c / m_{*}\right)$ replaced by $\exp \left(-c^{2} / m_{*}^{2}\right)$. In addition, the Lagrangian we discuss treats $u^{\mu}$ as a dynamical field.

Equation of State for Stringy Dark Energy. We define a dimensionless $\kappa=k / M_{\text {Planck }}$ scaled relative to the Hagedorn temperature which we designate as $M_{\text {Planck }}$. We further define the comoving momentum $c=\kappa / a(t)$ where $a(t)$ is the cosmological scale factor.

The dispersion relation in [1] is linear for small $k, \omega(c)^{2} \sim c^{2}$ as is necessary to reproduce scaleinvariant large-scale fluctuations as well as the black-body CMB pectrum. At very high $c, \omega(c)$ falls as a gaussianl. We parametrize these features by the form

$$
\omega(c)=c e^{-\gamma c^{2}}
$$

where $\gamma \sim O(1)$ is real.

To estimate the equation of state, we find it most convenient to employ the formalism of general relativity in a preferred frame as is necessary, for example, to underwrite the non-gravitational theories [3] of Lorentz violation.

We adopt an action:

$S=\int d^{4} x \sqrt{-g}\left[R+\mathcal{L}_{u}+\lambda\left(u_{a} u^{a}-1\right)+\mathcal{L}_{\phi}\right]$

with

$$
\mathcal{L}_{\phi}=\partial_{\mu} \phi \partial^{\mu} \phi+\sum_{n=1}^{\infty} \frac{(-\gamma)^{n}}{n ! M_{\text {Planck }}^{2 n}} D^{2 n} \phi D^{2} \phi
$$

The four terms in $S$ require further explication. The first is the Einstein-Hilbert action. In the second,

\footnotetext{
${ }^{1}$ Note that in 11 an exponential $\omega(c)=c e^{-\gamma c}$ rather than a gaussian form $\omega(c)=c e^{-\gamma c^{2}}$ was discussed but the qualitative features of the putative dark energy candidate remain unchanged; we find the gaussian form more convenient to develop a covariant lagrangian formalism
} 
$u^{a}$ is a comoving timelike vector, specifying the preferred frame for FRW cosmology, necessarily promoted to the status of a field to allow consistent conservation laws. For $\mathcal{L}_{u}$ we may write, for example, a form similar to Eq.(12):

$$
\mathcal{L}_{u}=\sum_{n=0}^{\infty} \frac{(-\beta)^{n}}{n ! M_{\text {Planck }}^{2 n}} D^{2 n} u_{a} D^{2} u^{a}
$$

Note that Eq.(13) is merely illustrative and physically reasonable, although our conclusions do not depend on a specific choice for $\mathcal{L}_{u}$. It is important that we do not specialize to the Yang-Millslike term 4.5.5] $\mathcal{L}_{u} \propto F^{a b} F_{a b}$ with $F_{a b}=2 \nabla_{[a} u_{b]}$ since this would lead to the unacceptable result for $w=\langle P\rangle /\langle\rho\rangle$ found in [6. In our analysis the more general Eq. (13) is crucial in obtaining a physically-meaningful equation of state. In the third term of Eq.(11), $\lambda$ is a lagrange multiplier for the constraint that $u^{a}$ be of unit lengthad have squared norm. The fourth and last term in Eq.(11) is a choice for $\mathcal{L}_{\phi}$ which leads to the form of modified dispersion relation $\omega(c)=\operatorname{cexp}\left(-\gamma c^{2}\right)$. Here $D^{2} \phi=-D^{a} D_{a} \phi=-q^{a c} \nabla_{a}\left(q_{c}^{b} \nabla_{b} \phi\right)$ with $q_{a b}$ the spatial metric orthogonal to $u^{a}: q_{a b}=-g_{a b}+u_{a} u_{b}$.

The contributions to the stress-energy tensor are from $T_{a b}^{(u)}+T_{a b}^{(\phi)}$. We find now that the pressure $<P>=\frac{1}{3}<T_{i i}>$ receives a contribution from $T_{i i}^{(\phi)}$ from which we therefore derive the result:

$$
\begin{aligned}
<P>= & \frac{N}{3} \int \frac{d c c^{2}}{\omega(c)} n(c)\left[\omega(c)^{2}+2 \sum \frac{\left(\gamma c^{2}\right)^{n+1}}{n !}\right. \\
& \left.-2 \sum \frac{(n+1)\left(\gamma c^{2}\right)^{n+1}(-1)^{n+1}}{n !}\right]
\end{aligned}
$$

and a similar contribution from $T_{i i}^{(u)}$. We may rewrite the pressure as follows

$<P>=\frac{N}{3} \int_{c_{H}}^{\infty} c^{2} d c n(c)\left[c \omega^{\prime}(c)+u_{a} \bar{\omega}^{\prime a}(u)\right]$

Here $\bar{\omega}$ is defined as the differential of Eqs.(11) and (13). In Eq.(15), the factor $\frac{1}{3}$ arises from the spatial dimension and the group velocity $v_{\text {group }} \propto c d \omega / d c$ is occurring naturally. The notation is $\omega^{\prime}=d \omega / d c$ and $\bar{\omega}^{\prime a}=d \bar{\omega} / d u_{a}$. Our model has some superficial similarity to, but is quite different from, k-essence [6].

Now we need the density $<T_{00}>$ which receives contributions from $T_{a b}^{(\phi)}$ and $T_{a b}^{(u)}$ in the general case. One finds by a similar analysis to the above for pressure that the mean density is

$$
<\rho>=N \int_{c_{H}}^{\infty} c^{2} d c n(c)(\omega(c)+\bar{\omega}(u))
$$

We next confirm that Eq.(15) and Eq.(16) satisfy conservation of energy for general $\omega$ and $\bar{\omega}$, thus confirming that $\mathcal{L}_{u}$ as illustrated in Eq.(13), is not constrained. We assume $\alpha$ and $\beta$ are of order one and that the occupation numbers satisfy $n(c) \simeq n(u) \sim O(1)$. We compute the conservation law as follows:

$\rho=\frac{1}{a^{3}}(\omega+\bar{\omega}) \quad$ and $\quad P=\frac{1}{3 a^{3}}\left(c \omega^{\prime}+u_{a} \bar{\omega}^{\prime a}\right)$

satisfy $T d S=d E+P d V=0$, that is

$$
\frac{d}{d t}\left(\rho a^{3}\right)+P \frac{d}{d t}\left(a^{3}\right)=0
$$

providing that

$$
\dot{\omega}(c)=-H c \omega^{\prime} \quad \text { and } \quad \dot{\bar{\omega}}=-H u_{a} \bar{\omega}^{\prime a}
$$

Given this consistency condition, an arbitrary modified dispersion relation $\omega$ and arbitrary choice of $\bar{\omega}$ satisfy conservation of entropy.

In summary of the equation of state, we have:

$$
w=\frac{\frac{1}{3} \int_{c_{H}}^{\infty} d c c^{2} n(c)\left(c \omega^{\prime}(c)+u_{a} \bar{\omega}^{\prime a}(u)\right)}{\int_{c_{H}}^{\infty} d c c^{2} n(c)(\omega(c)+\bar{\omega}(u))}
$$

With the illustrative choice given earlier one has $c \omega^{\prime}=\left(1-2 \gamma c^{2}\right) \omega$ and $u_{a} \bar{\omega}^{\prime a}=\left(1-2 \beta u^{2}\right) \bar{\omega}$ so for this case if $\beta<a^{2} / 2$ there is a positive contribution to pressure from the $u$ term and, as explained above, a negative pressure expected to dominate physically from the $c$ term.

In the absence of an explicit non-adiabatic computational framework let us assume that $w<-1$ but is not unacceptably-negative. The important and attractive point is that the order-of-magnitude estimate for the dark energy density giving $\Lambda \simeq$ $10^{-120}$ in Planckian units [1] is preserved even when we arrive at a sensible equation of state which, from the form of Eq.(20), still satisfies $w<-1$. A particular solution which asymptotes to $w=-1$ is discussed in [8].

Let us now consider such a case with $w<-1$. As an interesting example consider the case where the stringy dark energy density $\rho_{D E}$ increases with $(1+Z)^{-1}$ corresponding to $w=-\frac{4}{3}$. We note that a different theory predicting $w<-1$, based on a quantized very-light free scalar field, was considered in [9] and that the observational status of $w<-1$ was discussed in [10].

Transition from Deceleration to Acceleration. It is suggested by the study of Supernova 1997ff 11] 
that the present accelerated expansion of the Universe with deceleration parameter $q_{0}<0$ commenced at a finite transition redshift $Z_{t r}$ when $q\left(Z_{t r}\right)=0$. Another key red-shift is $Z_{e q}$ when the dark energy begins to dominate the total energy density; assuming flatness $\Omega_{\Lambda}(Z)+\Omega_{M}(Z)=1$, there was equality $\Omega_{\Lambda}\left(Z_{e q}\right)=\Omega_{M}\left(Z_{e q}\right)=1 / 2$.

We adopt the most favored values for the present matter and dark energy content in terms of the critical density $\rho_{c r}=\left(3 H_{0}^{2} / 8 \pi G\right)$ as $\left(\Omega_{D E}\right)_{0} /\left(\Omega_{M}\right)_{0}=$ 2 . These are suggested by the common intersection of three sources: the supernovae type IA data 12, 13, the observations of the acoustic peaks in the Cosmic Microwave Background from BOOMERANG 14 and MAXIMA [15 reviewed in [16] and the estimates of dark matter in large scale studies. For an overview, see [17]. The values of $Z_{t r}$ and $Z_{e q}$ satisfy

$$
\begin{gathered}
1+Z_{t r}=\left(-(1+3 w) \frac{\left(\Omega_{D E}\right)_{0}}{\left(\Omega_{M}\right)_{0}}\right)^{-1 / 3 w} \\
1+Z_{e q}=\left(\frac{\left(\Omega_{D E}\right)_{0}}{\left(\Omega_{M}\right)_{0}}\right)^{-1 / 3 w}
\end{gathered}
$$

where $w=<p>/<\rho>$ for the dark energy. Putting in $w=-4 / 3$ as in our example predicts $Z_{t r}=0.57$ and $Z_{e q}=0.19$. For comparison, these values for a cosmological constant are $Z_{t r}=0.59$ and $Z_{e q}=0.26$ while for quintessence models with $w>-1$ the corresponding $Z_{t r}, Z_{e q}$ values are different again.

From data on radio galaxies [18] there is some preliminary indication that $w<-1$ might be favored. Also, a global fit to several cosmological data sets gives [19] a range $-2.68<w<-0.78$ which implies that $w<-1$ is still a viable possibility, This inequality may be the first support for a stringy origin to the puzzling phenomenon of dark energy.

I wish to thank L. Mersini and L. Parker for discussions. This work was supported in part by the Office of High Energy, US Department of Energy under Grant No. DE-FG02-97ER41036.

[1] M. Bastero-Gil, P.H. Frampton and L. Mersini. hep-th/0110167. Phys. Rev. D65 106002 (2002).
[2] L. Mersini, M. Bastero-Gil and P. Kanti, Phys. Rev. D64, 043508 (2001).

[3] S. Coleman and S.L. Glashow, Phys. Lett. 405B, 249 (1997); Phys. Rev. D59, 116008 (1999).

D. Colladay and V.A. Kostelecky, Phys. Rev. D55, 6760 (1997); idem, D58, 116002 (1998).

[4] T. Jacobson and D. Mattingly, Phys. Rev. D63, 041502 (2001).

[5] T. Jacobson and D. Mattingly, Phys. Rev. D64, 024028 (2001).

[6] M. Lemoine, M. Lubo, J. Martin and J.-P. Uzan, Phys. Rev. D65, 023510 (2002).

[7] C. Amendariz-Picon, V. Mukhanov and P.J. Steinhardt, Phys. Rev. Lett. 85, 4438 (2000). astro-ph/0004134. Phys. Rev. D63, 103510 (2001).

[8] M. Bastero-Gil and L. Mersini. astro-ph/0205271

[9] L. Parker and A. Raval, Phys. Rev. Lett. 86, 749 (2001).

[10] R.R. Caldwell. astro-ph/9908168.

[11] A.G. Riess et al. Astrophys. J. 560, 49 (2001). astro-ph/0104455.

[12] G. Goldhaber et al. Nucl. Phys. Proc. Suppl. 38, 435 (1995). A. Goobar and S. Perlmutter. astro-ph/9505022. S. Perlmutter et al. Astrophys. J. 483, 565 (1997). astro-ph/9608192; Nature. 391, 51 (1998) astro-ph/9712212 Astrophys. J. 517, 565 (1999). astro-ph/9812133; Int. J. Mod. Phys. A 15S1, 715 (2000). A. Goobar et at. Phys. Scripta T85, 47 (2000).

[13] P.M. Garnevich et al. Astrophys. J. 493, L53 (1998). P. Schmid et al. Astrophys. J. 507, 46 (1998). astro-ph/9805200. A.G. Riess et al. Astrophys. J. 116, 1009 (1998). astroph-9805201. P.M. Garnevich et al. Astrophys. J. 509, 74 (1998). astro-ph/9806396. A.V. Filippenko and A.G. Riess. Phys. Rept. 307, 31 (1998). astro-ph/9807008.

[14] P.D. Mauskopf et al. Astroph. J. 536, L59 (2000). astro-ph/9911444. A. Melchiorri et al. Astrophys. J. 536, L63 (2000). astro-ph/9911445. A. Lange et al. Phys. Rev. D63, 042001 (2001). astro-ph/0005004. A.H. Jaffe et al. Phys. Rev. Lett. 86, 3475 (2001). astro-ph/0007333. C.B. Netterfield et al. astro-ph/0104460.

[15] See e.g. J.R. Bond et al. astro-ph/0011378.

[16] M. Kamionkowski and A. Kosowsky, Ann. Rev. Nucl. Part. Sci. 49, 77 (1999). astro-ph/9904108. W. Hu, M. Fukugita, M. Zaldarriaga and M. Tegmark, Astrophys. J. 549, 669 (2001). astro-ph/0006436.

[17] N.A. Bahcall, J.P. Ostriker, S. Perlmutter and P.J. Steinhardt, Science 284, 1481 (1999).

[18] R. Daly, M.P. Mory and E.J. Guerra. astro-ph/0203113.

[19] S. Hannestad and E. Mörtsell. astro-ph/0205096. 